\title{
Endoscopic Excision of Juvenile Nasopharyngeal Angiofibroma: A Case Series
}

\author{
Raji A, Dbab L, Ait el abdia R, Rochdi Y, Nouri H and ELfakiri M \\ ENT Department, Mohammed VI University Hospital, Marrakech, Morocco
}

*Corresponding author: Dbab L, ENT Department, Mohammed VI University Hospital, Marrakech, Morocco. Tel: 212677359627, E-mail: lamiadbaborl@gmail.com

Citation: Raji A, Dbab L, Ait el abdia R, Rochdi Y, Nouri H, et al. (2018) Endoscopic Excision of Juvenile Nasopharyngeal Angiofibroma: A Case Series. J Otolaryngol Res 1: 101

Article history: Received: 6 November 2017, Accepted: 22 January 2018, Published: 24 January 2018

\section{Abstract}

Objectives: To present our experience of excision of juvenile nasopharyngeal angiofibroma (JNA) through endoscopic approach.

Method: Retrospective case series review of six cases of JNA patients, encountered between 2010 and 2017. All patients underwent endoscopic excision, preceded by embolisation.

Results: All patients were male presenting with typical complaints of progressive unilateral nasal blockage, spontaneous painless epistaxis hyposmia and sleep disturbance. The patients' presentation comprised of IA to IIC stages based on Radkowski classification, with one recurrence case. Maxillary artery was the main blood supply to the lesion. Surgical approach applied was endoscopic. All patients were discharged well with no major complications, nor cases of recurrence.

Conclusion: Endoscopic approach is possible for varies stages of JNA. Preoperative angiographic embolisation has minimised the blood loss intraoperatively. The endoscopic method has reduced the postoperative morbidity.

Keywords: Juvenile nasopharyngeal angiofibroma (JNA); Embolisation; Endoscopic approach

\section{Introduction}

Juvenile Nasopharyngeal Angiofibroma (JNA) is a rare, benign, and highly vascular and locally aggressive tumor that accounts for $0.05 \%$ to $0.5 \%$ of all head and neck neoplasms generally seen in prepubertal and mainly targeted to male adolescence [1-6]. The tumor originates from the superior edge of the sphenopalatine foramen and advances submucosally, through natural ostia, along canals and nerves and by bone erosion to the infratemporal fossa, pterygoid canal, parasellar region, sphenoid basis and the orbit [1,4-6]. Facial deformity, reduced vision, exopthalmos and opthalmoplegia may develop. Therefore, despite its benign nature, JNA should be treated as radically as possible to prevent recurrence which is reported to be between 23 and 27.5\% [1,5]. Several staging classification has been proposed, however Radkowski classification has been widely used for its practicality (Table 1) [3-5,7].

\begin{tabular}{|c|c|}
\hline IA & Limited to the nose and/or nasopharynx \\
\hline IB & Same as IA but with extension into one or more paranasal sinuses \\
\hline IIA & $\begin{array}{l}\text { Minimal extension through the sphenopalatine foramen, into } \\
\text { and including a minimal part of the Medial-most part of the } \\
\text { pterygomaxillary fossa }\end{array}$ \\
\hline IIB & $\begin{array}{l}\text { Full occupation of the pterygomaxillary fossa, displacing the the } \\
\text { posterior wall of the maxillary antrum forward. Lateral and/or } \\
\text { anterior displacement of branches of the maxillary artery. Superior } \\
\text { extension may occur, eroding orbital bones }\end{array}$ \\
\hline IIC & $\begin{array}{l}\text { Extension through the pterygomaxillary fossa into the cheek and } \\
\text { temporal fossa or posterior to pterygoid plates }\end{array}$ \\
\hline IIIA & Erosion of skull base-minimal iwntracranial extension \\
\hline IIIB & $\begin{array}{c}\text { Erosion of skull base-extensive intracranial extension with or } \\
\text { without cavernous sinus }\end{array}$ \\
\hline
\end{tabular}

Table 1: Staging of JNA according to Radkowski, et al. [7] 
Surgical resection remains the mainstay of treatment. The development of embolization methods and endoscopic techniques had a significant impact on this evolution [10].

The purpose of this study was to present our experience with the endoscopic management of JNA using retrospective chart review of six male patients (mean age 15.7 years) with JNA who were treated endoscopically at our institution between the years 2010 and 2017.

\section{Materials and Methods}

This is a retrospective review of six patients with JNA cases presented to our centre from 2010 till 2017. The demographic data, clinical presentations, clinical findings, imaging investigations and treatment modalities of these patients were collected and reviewed based on medical records. All patients had preoperative imaging including computed tomography (CT scan) magnetic resonance imaging (MRI) and angiography (Figure 1a and 1b).

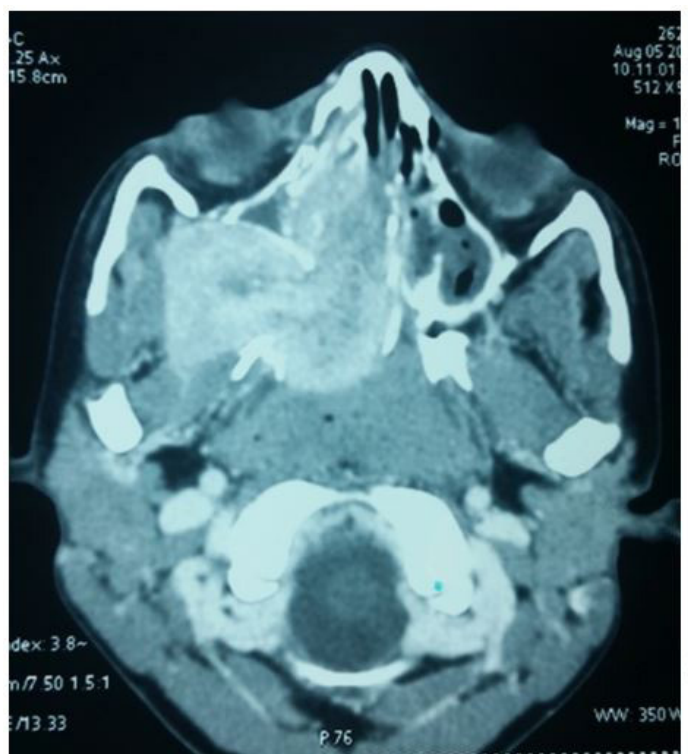

(a)

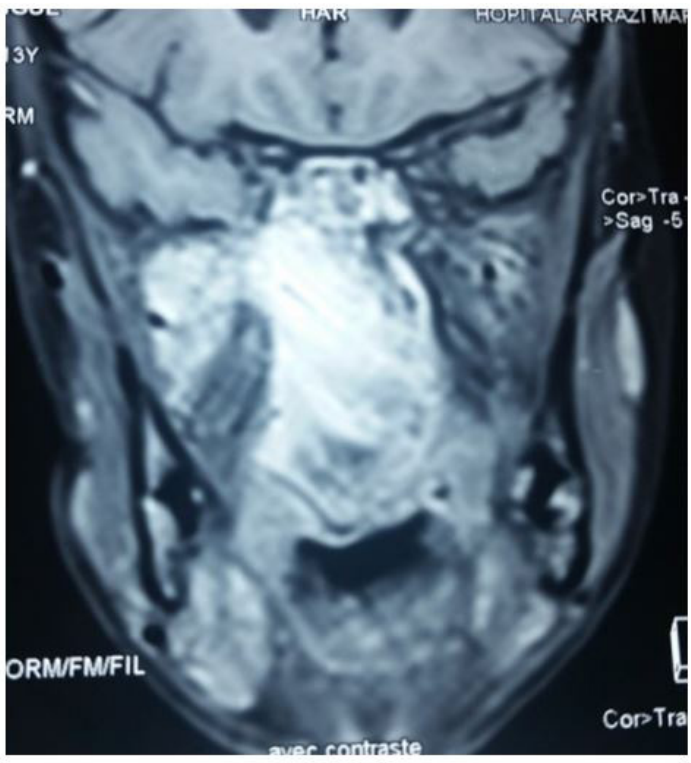

(b)

Figure 1: (a) Axial CT scan of a 21-year-old patient with JNA stage IIc. The lesion occupies the nasopharynx and penetrated the right pterygopalatine fossa (open arrows) through an enlarged sphenopalatine foramen

(b) Coronal T1-weighted MRI scan of the same patient. The enhancing lesion invades into the right infratemporal fossa

The classification was used to classify all tumors based on the patients' CT scan and MRI reports according to the Radkowski's classification. Preoperative embolisation within 48 hours of surgery was performed to all patients (Figure 2).

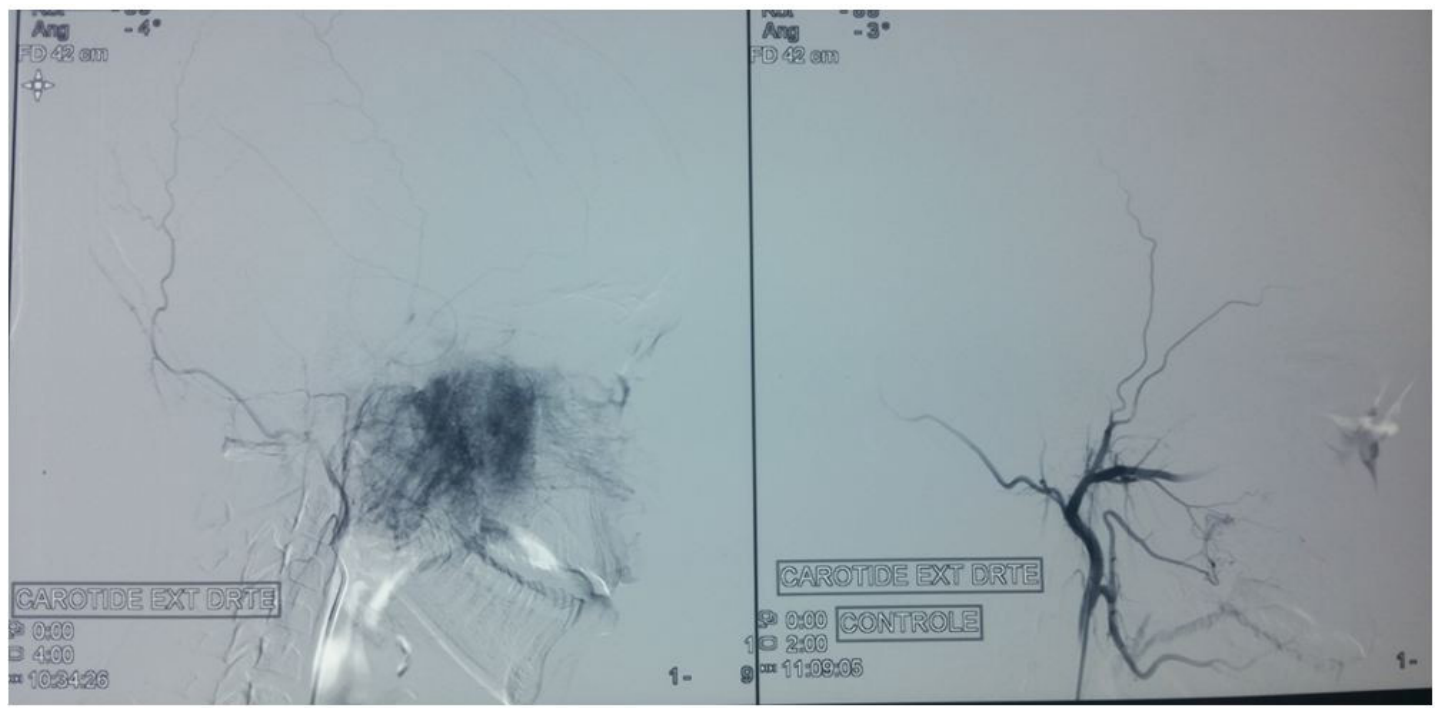

Figure 2: Angiography pre-embolisation (on the right): Shows tumour blush from the sphenopalatine artery via the internal maxillary artery and postembolisation (on the left) images of the right external carotid artery: shows no visible tumour blush 
The operations were performed under general anesthésia. In all cases, the final histopathological examination revealed juvenile angiofibroma. The modality of treatment for all six patients was transnasal endoscopic approach. Five of these cases were primary resections except one, was a case of recurrence.

\section{Results}

There were six patients treated with endoscopic approach for JNA in our centre from 2010 till 2017. All patients were male. The mean age of diagnosis was 16 (range 11-29) years old. Four of these patients presented with spontaneous painless intermittent epistaxis (40\%), while two patients presented with profuse bleeding requiring blood transfusion (20\%). Other symptoms included progressive nasal blockage (20\%), reduction in sense of smell (10\%) and sleep disturbance (10\%).

Nasal endoscopy examination of nasal cavity revealed a vascular nasal mass mainly occupying the nasopharynx in all patients.

No nasal biopsies were taken from any of these patients. Contrast-enhanced CT imaging examination was ordered. Classification was used to stage the patients based on CT imaging result. Two patients $(33.3 \%)$ were at stage IA, one patient (16.7\%) was stage IIA, two patients (33.3\%) were stage IIB and one patient (16.7\%) was stage IIC. Four patients (66.7\%) presented with JNA arising from the left while two patients (33.3\%) raised from the right. All patients underwent angiography imaging and showed all tumors received blood supply mainly (80-90\%) from ipsilateral maxillary artery with some blood supply from the contralateral. Only one tumor had sole ipsilateral blood supply. The feeding vessels were embolised $48 \mathrm{~h}$ before surgery with no complications (Figure 2). Nasal packing remained for an average of 2.5 (range 2-4) days after surgery. No patient needed intensive care unit care postoperatively. Average duration of hospital stay for these patients was 6.3 (range 4-10) days. All patients were followed up for minimal of one year and no recurrences were noted.

\section{Discussion}

The radical external approaches are the mainstay of treatment for JNAs of any extent. They provide ample access to the tumor and space for surgical manipulations. Profuse bleeding, a hallmark of JNA resection, can be controlled easily by the surgeon who has both hands free to use suction and diathermy. The external scar of a lateral rhinotomy can be inconspicuous if the incision is closed meticulously or avoided at all if a mid facial degloving approach is adopted. The transoral Le Fort I approach provides wide exposure of the postnasal space and pterygopalatine and infratemporal fossa bilaterally. If there is large intracranial extension of JNA or high risk of severing the internal carotid artery or the cavernous sinus intraoperatively, a craniofacial approach is employed $[1,4,9]$. The morbidity of the external approaches in the treatment of JNA is well documented. Blood loss due to the extensive osteotomies for access may necessitate blood transfusions with all the associated risks of disease transmission [1]. Additionally, osteotomies increase the operative time and may interfere with the normal facial growth of the adolescent patient $[1,8,9]$. Cerebrospinal fluid leak, facial and infraorbital nerve damage, lacrimal dysfunction, facial deformities and dental malocclusion have been reported with transfacial approaches [1]. The lateral approach to the infratemporal fossa may result in trismus, hypesthesia and conductive hearing loss [1]. Endoscopic resection of nasal tumors with many advantages: bone resection is minimized, blood loss is reduced and the normal growth of the adolescent facial skeleton is not altered significantly [4,7]. By the endonasal access, the scar and the manipulation of the soft tissues of the anterior face are avoided. The operative time and hospital stay are therefore reduced. Another advantage of the endoscopic resection of JNA is the improved, multiangled and magnified view of the tumor limits which facilitates tumor resection $[5,7,8,10,11]$.

The limits for endoscopic resection are extensive intracranial extension to middle cranial fossa or extension lateral to cavernous sinus or optic nerve. In these tumors endoscopic approach is not justified and combined approaches or radiotherapy can be the treatment of choice [8].

This type of surgery also seems to decrease the rate of recurrence as fingerlike extensions of tumor can be precisely seen with endoscopes [7]. The recurrence rate of $10 \%$ is lower than the values reported for the external surgical approach (14.5\%). Thus, the endoscopic technique is at least as good as the external technique regarding the recurrence rate [7].

\section{Conclusion}

In conclusion, the endoscopic treatment is an evolving treatment modality of JNA, and its limits are progressively pushed further. Also endoscopic approach for JNA has permits less post-operative morbidity; improve visualisation compare to traditional open methods, and lower recurrence. It is obvious that endoscopic surgery has increased our vision and range of our operations.

\section{References}

1. Fyrmpas G, Konstantinidis I, Constantinidis J (2012) Endoscopic treatment of juvenile nasopharyngeal angiofibromas: our experience and review of the literature. Eur Arch Otorhinolaryngol 269: 523-9.

2. Boghani Z, Husain Q, Kanumuri VV, Khan MN, Sangvhi S, et al. (2013) Juvenile nasopharyngeal angiofibroma:a systematic review and comparison of endoscopic, endoscopic assisted, and open resection in 1047 cases. Laryngoscope.123: 859-69.

3. Latoo MA, Ul Hamid W, Jallu AS, Ul Islam M, Pampori RA (2017) Nasopharyngeal Angiofibroma: Paradigm Shift in Management. Indian J Otolaryngol Head Neck Surg 69: 47-51. 
4. Khoueir N, Nicolas N, Rohayem Z, Haddad A, Abou Hamad W (2014) Exclusive endodcopic resection of juvenile nasopharyngeal angiofibroma: a systematic review of the literature. Otolaryngol Head Neck Surg 150: 350-8.

5. Mørkenborg ML, Frendø M, Stavngaard T, Von Buchwald C (2015) Bilateral independent juvenile nasopharyngeal angiofibroma: case report.J Laryngol Otol 129: $1032-5$.

6. Makhasana JA, Kulkarni MA, Vaze S, Shroff AS (2016) Juvenile nasopharyngeal angiofibroma. J Oral Maxillofac Pathol 20: 330.

7. Lim LY, Irfan Mohamad A, Tang IP (2016) Endoscopic excision of juvenile nasopharyngeal angiofibroma: A case series. Egypt J ENT All Sci 18: 173-6.

8. Onerci TM, Yücel OT, Oğretmenoğlu O (2003) Endoscopic surgery in treatment of juvenile nasopharyngeal angiofibroma. Int J Pediatr Otorhinolaryngol 67: 1219-25.

9. Iovanescu G, Ruja S, Cotulbea S (2013) Juvenil nasopharngeal angiofibroma:Timisora ENT Department's experience. Int J Pediatr Otorhinolaryngol 77: 1186-9. 10. Kopeć T, Borucki Ł, Szyfter W (2014) fully endoscopic resection of juvenile nasopharyngeal angiofibroma own experience and clinical outcomes. Int J Pediatr Otorhinolaryngol 78: 1015-8.

11. Huang J, Sacks R, Forer M (2009) Endoscopic resection of juvenile nasopharyngeal angiofibroma. Ann Otol Rhinol laryngo.118: 764-8. 